\title{
NUMERICAL STUDY ON CHARACTERISTICS OF FLOW AND THERMAL FIELDS AROUND ROTATING CYLINDER
}

\author{
Kamel Korib*, Mohamed Roudane, Yacine Khelili \\ Department of Mechanical Engineering, University Saad Dahlab, Blida 1, Algeria
}

Received 20.10.2019

Accepted 27.02.2020

\begin{abstract}
In this paper, a numerical simulation has been performed to study the fluid flow and heat transfer around a rotating circular cylinder over low Reynolds numbers. Here, the Reynolds number is 200 , and the values of rotation rates $(\alpha)$ are varied within the range of $0<\alpha<6$. Two-dimensional and unsteady mass continuity, momentum, and energy equations have been discretized using the finite volume method. SIMPLE algorithm has been applied for solving the pressure linked equations. The effect of rotation rates $(\alpha)$ on fluid flow and heat transfer were investigated numerically. Also, time-averaged (lift and drag coefficients and Nusselt number) results were obtained and compared with the literature data. A good agreement was obtained for both the local and averaged values.
\end{abstract}

Keywords: unsteady flow; rotation rates; circular cylinder; Reynolds number.

\section{Introduction}

Cross-flow normal to the axis of a stationary circular cylinder and the associated problems of heat and mass transport are encountered in a wide variety of engineering applications. Both experimental measurements and numerical computations have confirmed the onset of instability of the wake flow behind a cylinder beyond a critical Reynolds number.

Zdravkovich [1] has compiled almost all the experimental, analytical and numerical simulation data on flow past cylinders, available since 1938 and systematically classified this challenging flow phenomenon into five different flow regimes based on the Reynolds number. In the present study, the computation is restricted only to the first few regimes designated by Zdravkovich as (1) creeping laminar state (L1) of flow ( $0<\mathrm{Re}<$ 4 ), (2) laminar flow (L2) with steady separation $(4<\mathrm{Re}<48$ ) forming a symmetric contra-rotating pair of vortices in the near wake, (3) laminar flow (L3) with periodic vortex shedding ( $48<\operatorname{Re}<180)$, and finally (4) part of the transition-in-wake $(\mathrm{TrW})$

*Corresponding author: Kamel Korib, kamelkorib@gmail.com 
regime $(180<\operatorname{Re}<400)$ when the three- dimensional instabilities lead to the formation of streamwise vortex structure.

Recently, Rajani et al. [2] have studied the laminar flow numerically past a circular cylinder for $\mathrm{Re}=100$ to 400 . An implicit pressure-based finite volume method was used here. The temporal evolution of the lift and drag coefficients have been computed separately from both 2-D and 3-D simulations of the flow for the values of $\operatorname{Re}=100,200$, 250, 300 and 400. They also reported that up to $\mathrm{Re}=200,2-\mathrm{D}$ and 3-D computation results are observed to be almost overlapping, showing no significant difference in the temporal variation of the lift and drag coefficients at the statistically stationary state.

In the study of wake dynamics, bluff body rotation has always drawn considerable attention, mainly due to its effects on boundary layer separation and the Magnus effect. An increase in the lift magnitude more than classic Prandtl's limit due to an increase in the rotation rate of the circular cylinder was proposed by Gaulart [3]. For the similar flow configuration, Kang and Choi [4] followed with the numerical solution of the unsteady governing equations in the primitive variables velocity and pressure for flows with $\mathrm{Re}=$ 60,100 and 160 with $0=q=2.5$. Their results showed that vortex shedding vanishes when q increases beyond a critical value which follows a logarithmic dependence on the Reynolds number (e.g., the critical dimensionless rotation rate $\mathrm{q}=1.9$ for $\mathrm{Re}=160$ ). Later, the work of (Mittal \& Kumar, 2003) performed a comprehensive numerical investigation by fixing a moderate value of $\mathrm{Re}=200$ while considering a wide interval for the dimensionless rotation rate of $0 \leq \mathrm{q} \leq 5$. They used the finite-element method to solve the unsteady incompressible Navier-Stokes equations in two-dimensions for the primitive variables velocity and pressure [5].

In work [6], the laminar flow and heat transfer from a rotating circular cylinder with uniform planar shear was investigated, where the free stream velocity varies linearly across the cylinder using Multi-Relaxation-Time (MRT) LBM. Recently, the convective heat transfer from a rotating cylinder with inline oscillation was studied in the literature [7] at Re numbers of 100, 200, and 300. Different rotational speeds of the cylinder (0$2.5)$ are considered at various oscillating amplitudes and frequencies with three different Pr numbers of $0.7,6$, and 20.

Paramane et al. [8] investigated the forced convection heat transfer numerically across a rotating circular cylinder in the 2-D laminar regime. They concluded that the rotation could be used as a drag reduction and heat transfer suppression technique. Subsequently, Paramane et al. [9] studied the free stream flow numerically and forced convection heat transfer across a rotating cylinder, dissipating heat flux for Reynolds numbers of 20-160 and a Prandtl number of 0.7. Their results show that, at higher rotational velocity, the Nusselt number is almost independent of Reynolds number and the thermal boundary conditions.

Experimental measurements of the flow past a rotating cylinder were performed by Barnes [10]. At low rotation, rates to determine the value at which shedding is suppressed for Reynolds numbers between 50 and 65. The vortex shedding behind a rotating cylinder disappears when $\alpha$ is increased above the value of 2 is showed by Stojkovic et al. [11].

Because there is a lack of study of flow over a rotating cylinder and due to its broad application, heat transfer from a circular cylinder with constant wall temperature is investigated by using numerical method. 


\section{Problem Statement and Mathematical Formulation}

Consider the two-dimensional, laminar flow of an incompressible Newtonian fluid with a uniform inlet velocity $U_{\infty}$ and temperature $T_{\infty}$ across an infinitely long (in the zdirection) circular cylinder (Fig. 1). A circular cylinder of diameter $D$ is placed concentrically in a circular domain of diameter $D_{\infty}$, to convert the physical problem into a computational equivalent. The radius of the enveloping circular domain is chosen to be sufficiently large in order to minimize the boundary effects.

The surface of the solid cylinder is maintained at a constant wall temperature, $T_{w}$. The thermophysical properties of the streaming liquid are assumed to be independent of the temperature and the viscous dissipation effects in the energy equation are neglected in this study.

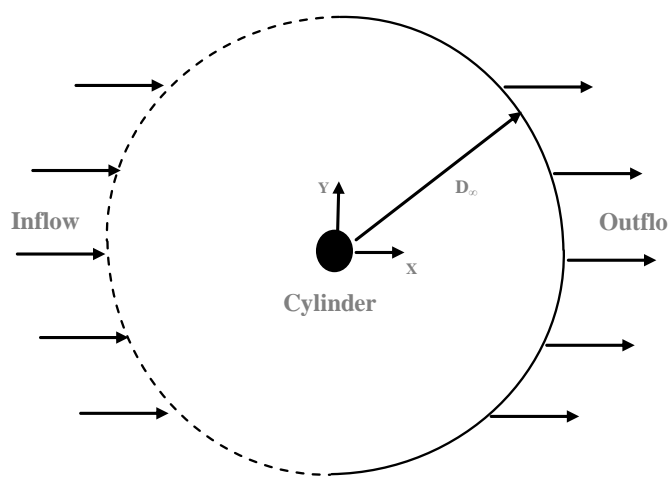

Fig. 1. Schematics of the unconfined flow around a circular cylinder.

\section{Governing equations}

The flow and heat transfer phenomena are governed by the continuity, NavierStokes and thermal energy equations written in their dimensionless forms, as follows. Continuity Equation:

$$
\frac{1}{r} \frac{\partial\left(r U_{r}\right)}{\partial r}+\frac{1}{r} \frac{\partial U_{\theta}}{\partial \theta}=0
$$

$\theta$-Momentum Equation:

$$
\frac{\partial U_{\theta}}{\partial t}+\frac{U_{\theta}}{r} \frac{\partial U_{\theta}}{\partial \theta}+U_{r} \frac{\partial U_{\theta}}{\partial r}+\frac{U_{r} U_{\theta}}{r}=-\frac{1}{r} \frac{\partial P}{\partial \theta}+\frac{1}{R e}\left(\frac{1}{r} \frac{\partial}{\partial r}\left(r \frac{\partial U_{\theta}}{\partial r}\right)+\frac{1}{r^{2}} \frac{\partial^{2} U_{\theta}}{\partial \theta^{2}}+\right.
$$

$\left.\frac{2}{r^{2}} \frac{\partial U_{r}}{\partial \theta}-\frac{U_{\theta}}{r^{2}}\right)$

$r$-Momentum Equation:

$$
\frac{\partial U_{r}}{\partial t}+\frac{U_{\theta}}{r} \frac{\partial U_{r}}{\partial \theta}+U_{r} \frac{\partial U_{r}}{\partial r}-\frac{U_{\theta}^{2}}{r}=-\frac{\partial P}{\partial r}+\frac{1}{R e}\left(\frac{1}{r} \frac{\partial}{\partial r}\left(r \frac{\partial U_{r}}{\partial r}\right)+\frac{1}{r^{2}} \frac{\partial^{2} U_{r}}{\partial \theta^{2}}-\right.
$$

$\left.\frac{2}{r^{2}} \frac{\partial U_{\theta}}{\partial \theta}-\frac{U_{r}}{r^{2}}\right)$ 
Energy Equation:

$$
\frac{\partial T}{\partial t}+\frac{U_{\theta}}{r} \frac{\partial T}{\partial \theta}+U_{r} \frac{\partial T}{\partial r}=\frac{2}{R e P r} \times\left(\frac{1}{r} \frac{\partial}{\partial r}\left(\frac{\partial T}{\partial r}\right)+\frac{1}{r} \frac{\partial}{\partial \theta}\left(\frac{1}{r} \frac{\partial T}{\partial \theta}\right)\right)
$$

\section{Boundary conditions}

According to Fig. 1 the governing Eqs. (1) - (4) are subjected to the following boundary conditions:

Inlet boundary $\left(r=R_{\infty}=150 R,-\pi / 2 \leq \theta \leq \pi / 2\right)$ :

$$
U_{\theta}=\alpha \sin \theta, U_{r}=-\alpha \cos \theta, T=T
$$

Outlet boundary $\left(r=R_{\infty}=150 R, \pi / 2 \leq \theta \leq-\pi / 2\right)$ :

$$
\frac{\partial U_{\theta}}{\partial r}=0, \frac{\partial U_{r}}{\partial r}=0, \frac{\partial T}{\partial r}=0
$$

Cylinder wall boundary $(r=R, 0 \leq \theta \leq 2 \pi)$ :

$$
U_{\theta}=0, U_{r}=0, T=T_{w}
$$

Auxiliary equations:

The wall pressure coefficient, $C_{P}$, may be defined as the following:

$$
C p=\frac{P-P_{0}+0.5 \rho U_{\infty}^{2}}{0.5 \rho U_{\infty}^{2}}
$$

Where $P_{0}$ is the pressure at the front stagnation point.

- In the time-periodic flow regime, there is also a net force acting on the cylinder in the lateral direction and this is expressed in terms of a lift coefficient $\mathrm{C}_{\mathrm{L}}$, defined as follows:

$$
C_{L}=\frac{F_{L}}{0.5 \rho D U_{\infty}^{2}}
$$

Where $F_{L}$ is the lift force.

- The local Nusselt number of the fluid, based on cylinder diameter, is defined as:

- non-dimensional rotation rate

$$
\alpha=\frac{\Omega D}{2 U_{\infty}}
$$

- The local Nusselt number of the fluid, based on cylinder diameter, is defined as:

$$
N u=-\left[\frac{\partial \theta}{\partial n}\right]_{\text {along the cylinder surface }}
$$

- Surface averaged Nusselt number of a fully developed thermal boundary layer is defined as:

$$
N u_{\text {ave }}=\frac{1}{s} \int_{s} N u d s
$$




\section{Grid generation}

In the present meshing scheme, a cylinder with a diameter $D$ resides in the center of the chosen computational domain. The outer boundary of the domain is circular with diameter $D_{\infty}$ from the center of the cylinder, Fig. 1 . The number of node points along the cylinder circumference and along the normal direction is represented by ' $N$ ' and ' $M$ ' respectively. We use $\mathrm{M}=144$ nodes stretched along the radial direction and $\mathrm{N}=121$ equispaced nodes around the circumferential direction. The normal wall distance of the first internal grid point is maintained at $\Delta \mathrm{r}=0.0001 \mathrm{D}$, required for adequate resolution of the sharp near-wall gradients of the flow variables.

\section{Numerical Method}

The present numerical investigation has been carried out using FLUENT (version 6.3.26). The unstructured quadrilateral cells of non-uniform grid spacing are generated using the commercial software GAMBIT (version 2.3.16). The grid is chosen to be sufficiently fine to capture the steep gradients in the vicinity of the cylinder. The secondorder upwind scheme has been used to discretize the convective terms in the momentum and energy equations. The SIMPLE scheme (semi-implicit method for the pressure linked equations) is used for solving the pressure-velocity decoupling. FLUENT solves the system of algebraic equations using the Gauss-Siedel $(\mathrm{G}-\mathrm{S})$ point-by-point iterative method.

\section{Results and Discussion}

\section{Validation of results}

In order to validate our numerical solution, estimated results for drag coefficients in forced convective heat transfer of fluid flow around a circular cylinder are compared with the available data in the literature. Separate runs were necessary to determine the results at specific Reynolds numbers of 100 and 200. Table 2 compares the drag coefficient $C_{D}$ obtained in the present research with those from references [2-17-18-1920-21]. The results are in excellent agreement with previous studies.

In Fig. 2, the stagnation pressure coefficient reduces from 1 as $\alpha$ increases. At $\alpha=$ 2 , when a closed streamline circulating around the cylinder can be observed, it becomes less than zero. For $\alpha \geq 2$, the pressure coefficient is negative everywhere on the cylinder rotation on the flow.

Table 1. Comparison between present results and literature data for at Re 100 and 200.

\begin{tabular}{lllll}
\hline Refrence & $\mathrm{Re}=$ & 100 & $\mathrm{Re}=$ & 200 \\
& $\mathrm{C}_{\mathrm{D}}$ & $\mathrm{St}$ & $\mathrm{C}_{\mathrm{D}}$ & $\mathrm{St}$ \\
\hline Ha. Roy [19] & 1.352 & 0.161 & 1.32 & 0.192 \\
I., M. S. [18] & 1.344 & 0.165 & 1.337 & 0.194 \\
Ding et al. [17] & 1.356 & 0.166 & 1.348 & 0.196 \\
B.N. Ra . [2] & 1.368 & 0.172 & 1.376 & 0.192 \\
Lam et al. [20] & 1.36 & 0.160 & 1.32 & 0.196 \\
M. and A. [21] & 1.368 & 0.172 & 1.376 & 0.192 \\
Present study & 1.322 & 0.163 & 1.316 & 0.183 \\
\hline
\end{tabular}




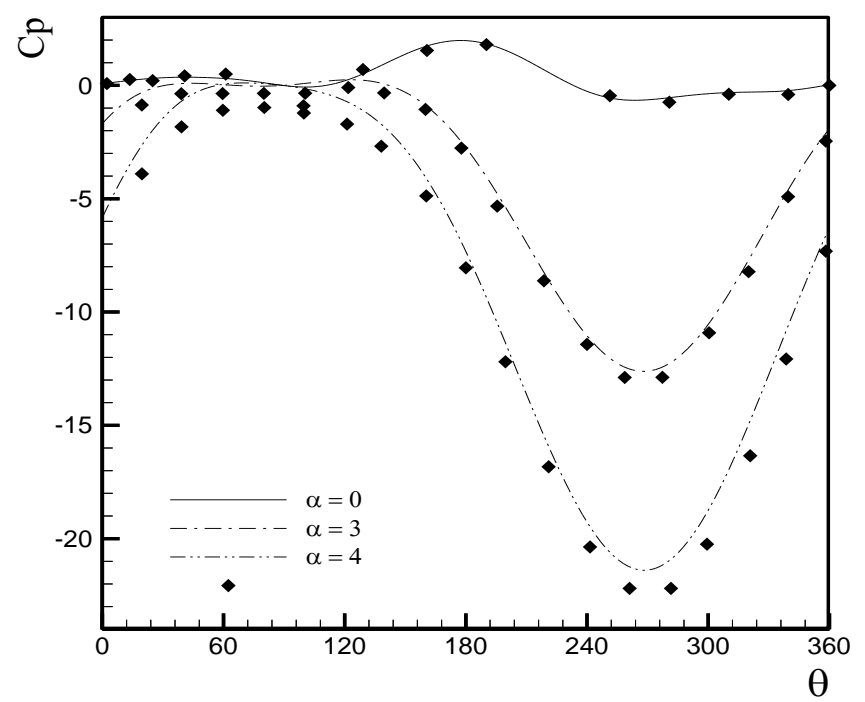

Fig. 2. Comparison results for pressure coefficient around the surface of the cylinder for various values of $\alpha$ at $R e=200$ with Mittal and Kumar (2003)

\section{Structure of flow field}

The time histories of the lift coefficient for the flow past a rotating cylinder for various values of $\alpha$ at $R e=200$ is presented in Fig. 3. The phase diagrams of $C_{L}$ and $C_{D}$ are shown in Fig. 4. For $0 \leq \alpha \leq 1.9$, a von Karman street is seen in the wake behind the cylinder; it achieves a steady-state for $\alpha>1.90$. An increase in the rotation rate is accompanied by an increased upward deflection of the wake and a reduction in its lateral width. At $\alpha=1.91$, the vortex shedding ceases and the flow achieves a steady state. It is seen that the flow remains steady for $1.91 \leq \alpha \leq 4.35$. However, the flow is unstable again for $4.34 \leq \alpha \leq 4.75$. Beyond $\alpha>4.75$, the flow is steady, but multiple solutions are observed. 


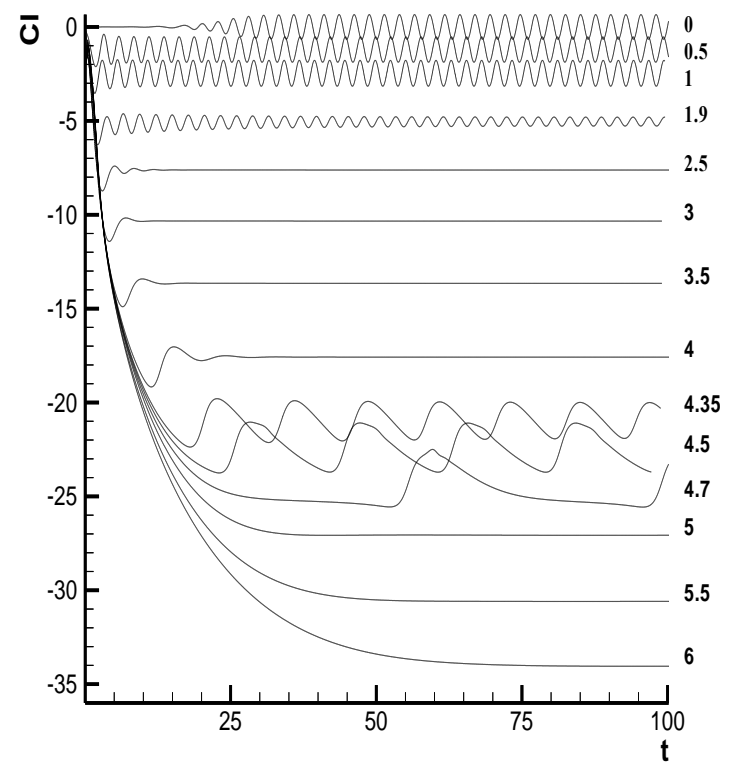

Fig 3. Temporal evolution of lift coefficients for laminar flow past a rotating circular cylinder at $\operatorname{Re}=200$.

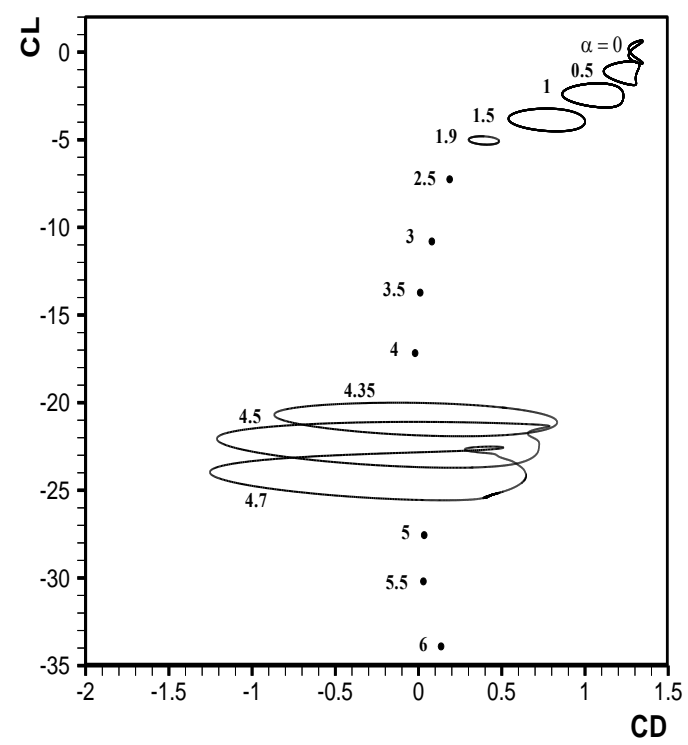

Fig. 4. Phase diagrams of $C_{L}$ and $C_{D}$ for various values of a for $R e=200$. 
The flow past a non-rotating cylinder is symmetric and characterized by two recirculation regions just behind the body. When increasing the rotation rate of the cylinder, the lower of these two regions disappears. The upper bubble, instead, detaches from the surface and becomes smaller. The stagnation point moves away from the cylinder surface rotating in the direction opposite to that of the cylinder rotation. Further increasing the value of $\alpha$, the upper vortex disappears and the rotation of the cylinder dominates flow. The critical $\alpha$ values are presented in Fig. 5 for different Reynolds numbers in comparison with the results of Stojkovic et al. (2003) and Mittal \& Kumar (2003). Fig 5 shows a stability map with the three curves representing the rotation rates ( $\alpha_{\mathrm{L} 1}, \alpha_{\mathrm{L} 2}$ and $\alpha_{\mathrm{L} 3}$ ) at which three transitions between steady and unsteady flows with the four flow regimes are found: I Unsteady regime for $\alpha \leq \alpha_{\mathrm{L} 1}$; I Steady regime at $\alpha_{\mathrm{L} 1} \leq \alpha \leq$ $\alpha_{\mathrm{L} 1}$; II Unsteady regime for $\alpha_{\mathrm{L} 2} \leq \alpha \leq \alpha_{\mathrm{L} 3}$; and II Steady regime for $\alpha>\alpha_{\mathrm{L} 1}$. Thus, for a $\operatorname{Re}$ in the vortex shedding regime of the stationary cylinder, the suppression of vortex shedding occurs at a specific rotation rate for a rotating cylinder and the flow remains steady at higher rotation rates except for a narrow range of $\alpha$ at which vortex shedding reappears.

Table 2 summarizes the basic integral parameters (Strouhal number $S t$, lift coefficient $C_{L}$, pressure lift coefficient $C_{L p}$, shear stress lift coefficient $C_{L f}$ and average Nusselt number), computed in the present study.

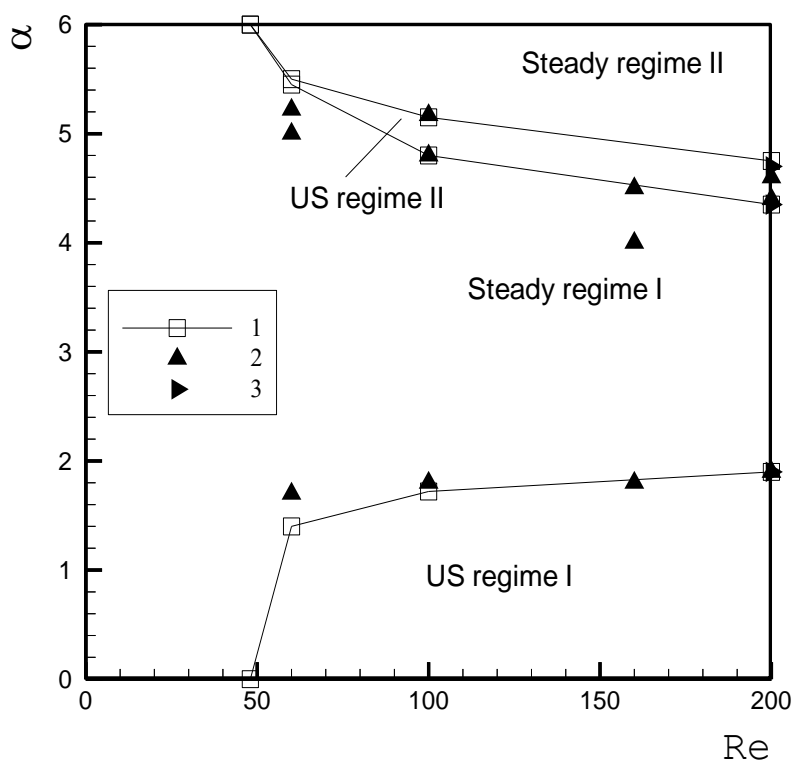

Fig. 5. Stability map for various Re and rotation rates $\alpha$. 1-present study, 2-results of [23], 3-results of the [5]. 
Table 2. Value of Strouhal number, mean lift coefficients, and average Nusselt number with $\alpha(\operatorname{Pr}=7.066)$.

\begin{tabular}{llllll}
\hline$\alpha$ & $S t$ & $C_{L}$ & $C_{L P}$ & $C_{L f}$ & Nuav \\
\hline 0 & 0.183 & 0.00 & 0.00 & 0.00 & 17.55 \\
0.5 & 0.184 & -0.5853 & -0.5917 & 0.006 & 17.03 \\
1 & 0.184 & -1.8337 & -1.7744 & -0.059 & 15.99 \\
1.5 & 0.187 & -3.2424 & -3.1046 & -0.138 & 13.27 \\
1.9 & 0.177 & -4.8073 & -4.5792 & -0.228 & 10.87 \\
2.5 & ---- & -7.6198 & -7.2841 & -0.336 & 9.38 \\
3 & ---- & -10.3379 & -9.9184 & -0.419 & 9.18 \\
3.5 & ---- & -13.6562 & -13.143 & -0.513 & 9.48 \\
4 & --- & -17.5829 & -16.967 & -0.616 & 9.49 \\
4.35 & 0.039 & -20.3172 & -19.653 & -0.664 & 8.89 \\
4.5 & 0.027 & -23.7087 & -22.942 & -0.767 & 6.50 \\
4.7 & 0.021 & -24.9829 & -24.177 & -0.806 & 5.39 \\
5 & ---- & -27.0707 & -26.235 & -0.836 & 3.73 \\
5.5 & ---- & -30.5923 & -29.691 & -0.901 & 3.16 \\
6 & ---- & -34.0467 & -33.087 & -0.959 & 2.82 \\
\hline
\end{tabular}

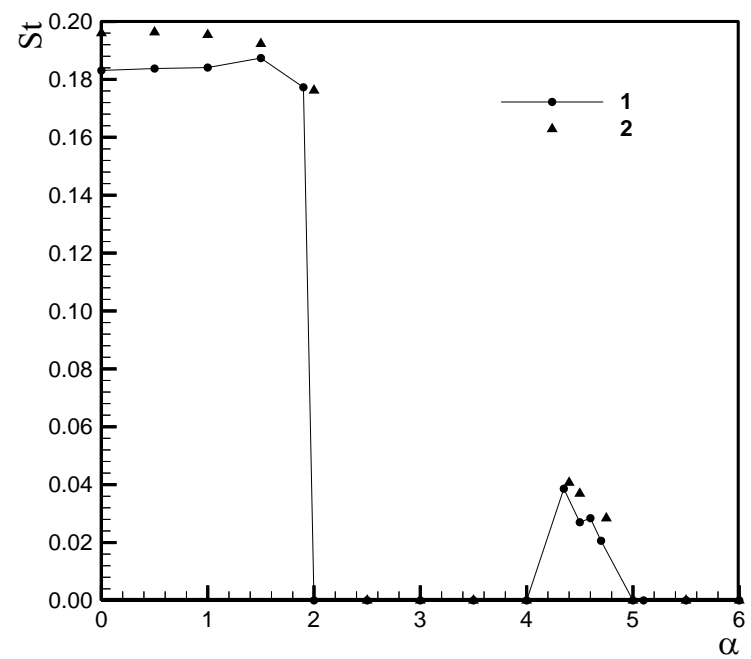

Fig. 6. Variation of Strouhal number with increasing rotation rate $\alpha$.

1 - present study, 2- results of [12].

Fig. 6 shows the variation of the Strouhal number for various rotation rate $\alpha$. The Strouhal number $(S t=f D / U)$ of vortex shedding is practically constant and decreases as a function of $\alpha$ before the first bifurcation, and is very low in the second mode interval. 
Fig. 7 shows the stagnation point for various rotation rates $\alpha$ (red line present the first stagnation points, and green line present the second stagnation points). It can be observed that for the case when $\alpha<3$, there are two apparent stagnation points.
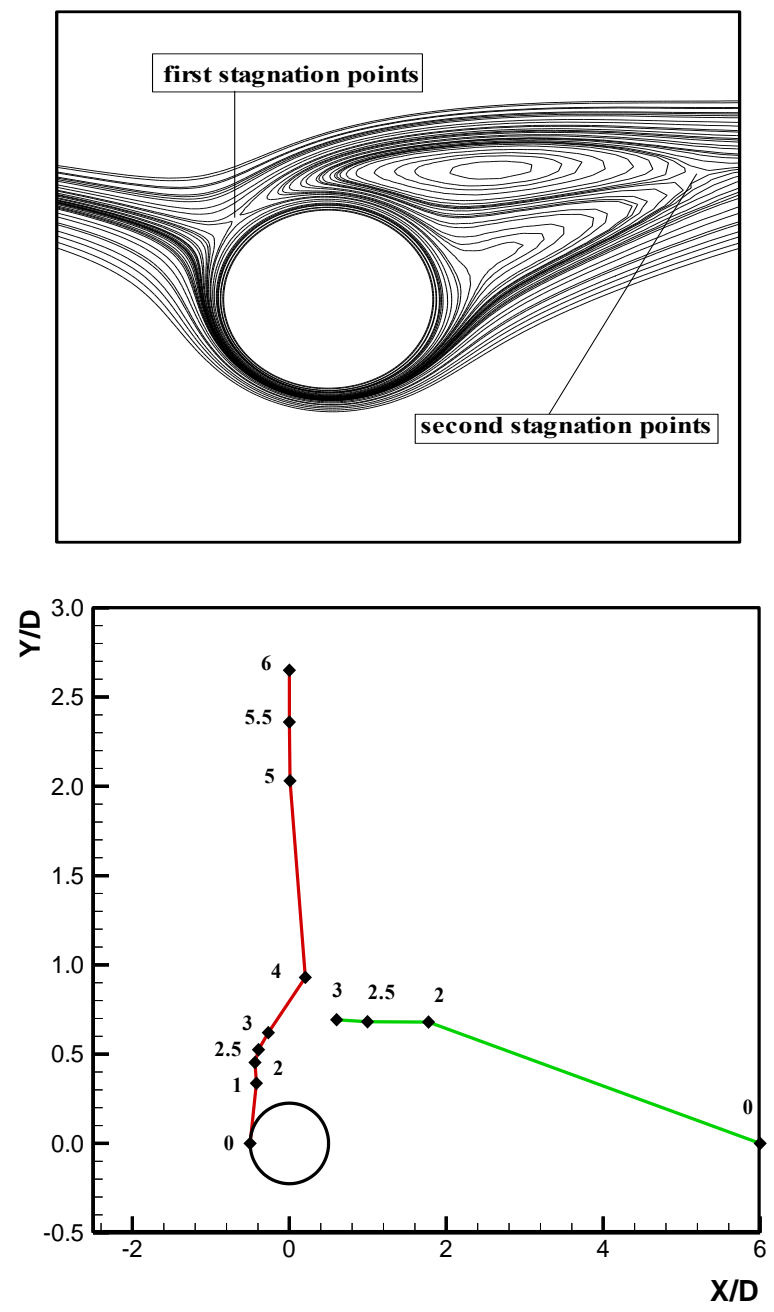

Fig. 7. Variation of stagnation point with increasing rotation rate $\alpha$.

The first is attributed to the collision of the rotating boundary layer with the freestream flow, while the second to the creation of a strong vortex downstream of the cylinder, which is formed due to the strong vorticity gradient between the free-stream layer and the rotating fluid, moving upstream and towards the top of the cylinder. As the dimensionless rotational rate increases, the upstream stagnation point moves downstream until $\alpha=4$. 
The downstream vortex begins to contract and entirely collapses at $\alpha=4$. For greater $\alpha$, the flow becomes swirling with a single stagnation point, which moves towards the outer region of the free-stream flow.

Fig. 8 shows that the maximum velocity $U_{\max }$ decreases as a function of $\alpha$ The thickness of the boundary layer $\mathrm{Y}$ at the point where the velocity is maximal is shown in Fig. 9, $\mathrm{Y}_{\max }$ increases with the rotation rate.

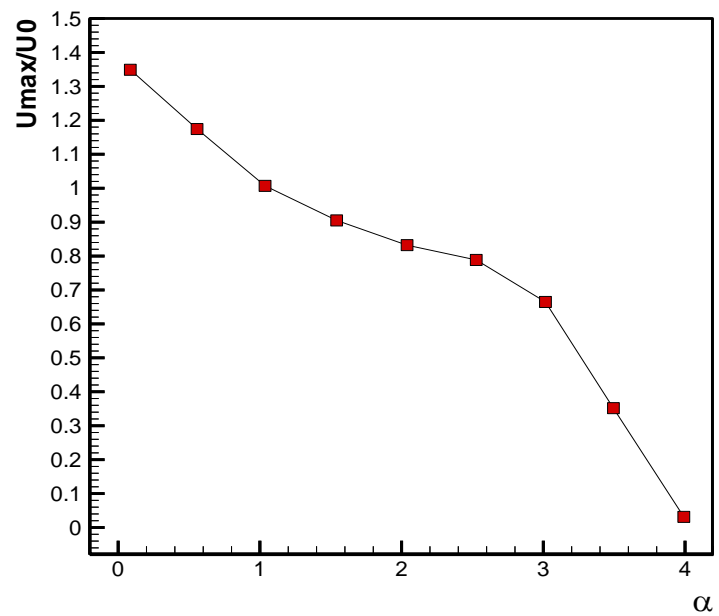

Fig. 8 Variation of the maximum velocity of the moyen fields as a function of $\alpha$.

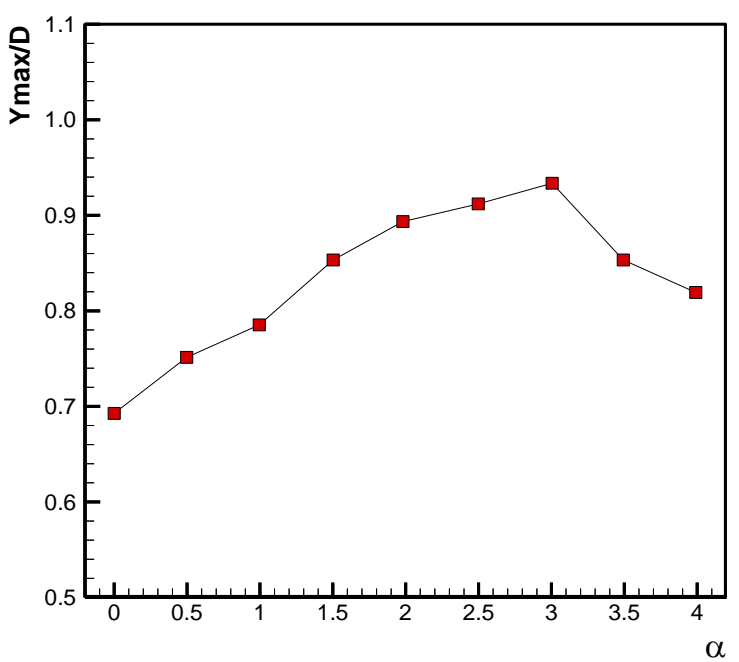

Fig. 9 Variation of the maximum boundary layer thickness of the moyen fields as a function of $\alpha$. 


\section{Heat transfer}

A comparison between local Nusselt number on the surface of the cylinder for different Reynolds numbers and various rotation rates $\alpha$ is shown in Fig. 10. For the stationary cylinder, the variation of $\mathrm{Nu}$ is found to be symmetrical at $\theta=180$; The maximum value of the local $\mathrm{Nu}$ number occurs at the front stagnation point $(\theta=0)$ for all $R e$. Further increase in rotation rate, $N u_{L}$ becomes almost independent of $R e$ at higher rotation rates $(\alpha=6)$.

Variation of average Nusselt number for different values of rotation rates $\alpha$ is shown in Fig. 11. For various rotation rates $\alpha$, to understand the suppression of heat transfer. It can be seen from this figure that the suppression increases with increasing $\alpha$, having a value of $65.18 \%$ for $R e=200$ at $\alpha=6$. Thus, cylinder rotation can be used not only for controlling flow but also as an efficient heat transfer suppression technique.

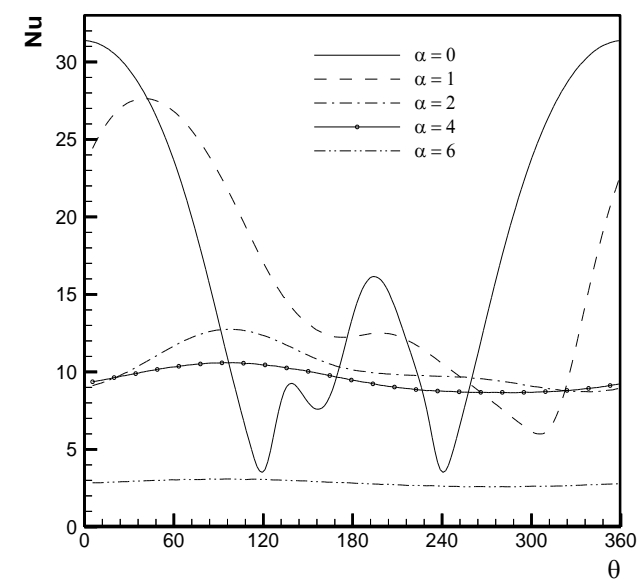

Fig 10. Variation of local Nusselt number on cylinder surface with various rotation rate $\alpha$.

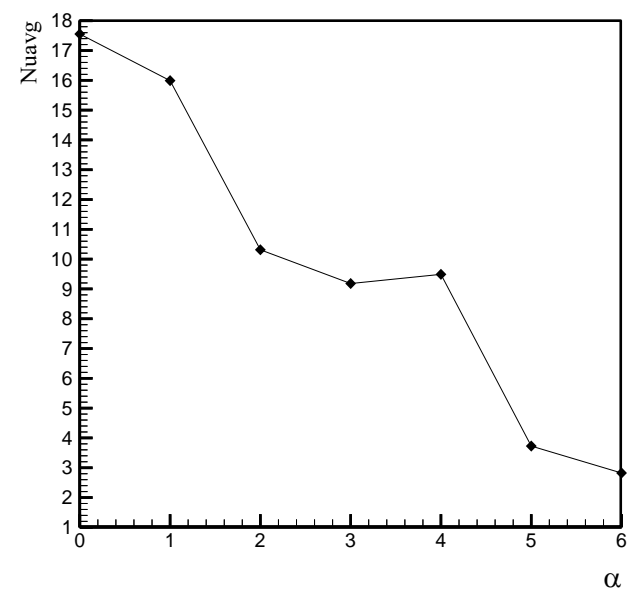

Fig. 11. Variation of average Nusselt number on the wall of cylinder versus various rotation rate $\alpha$. 

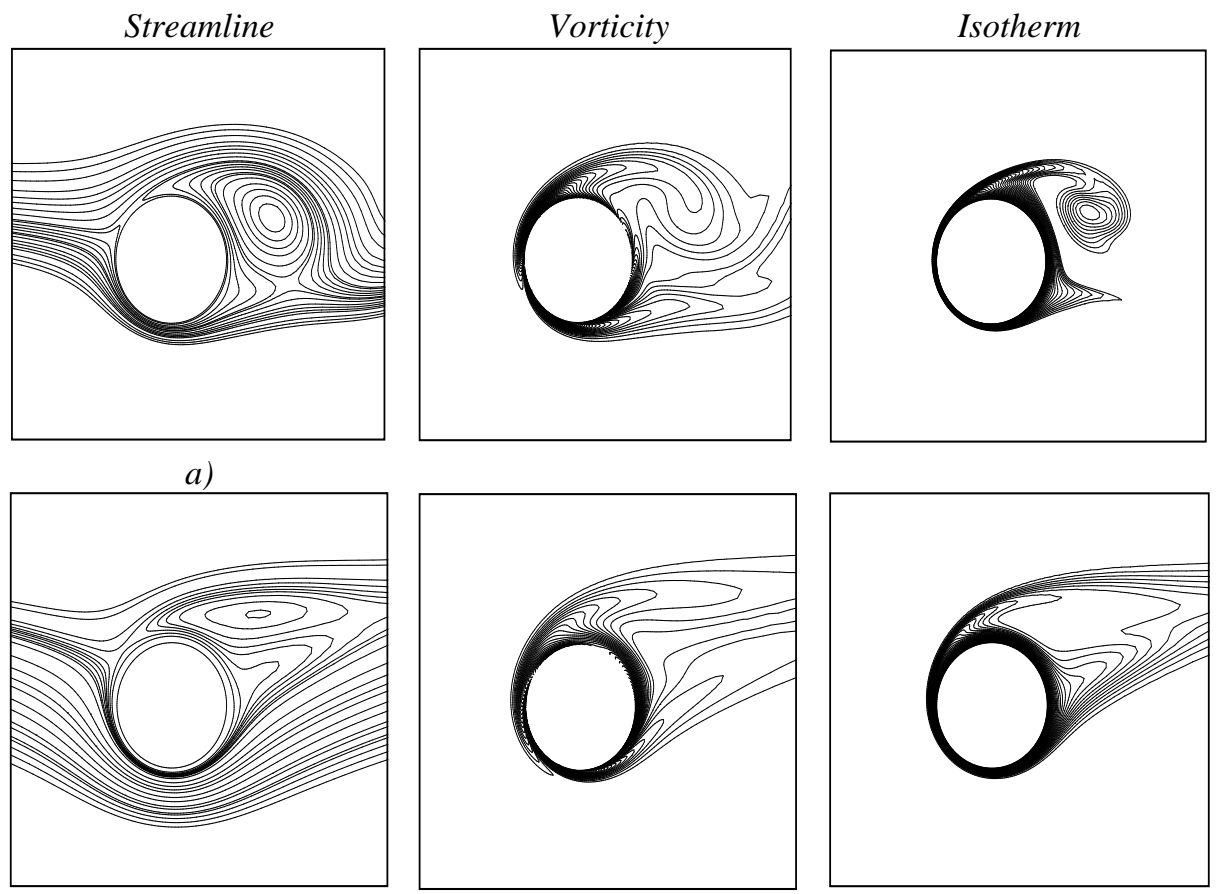

b)
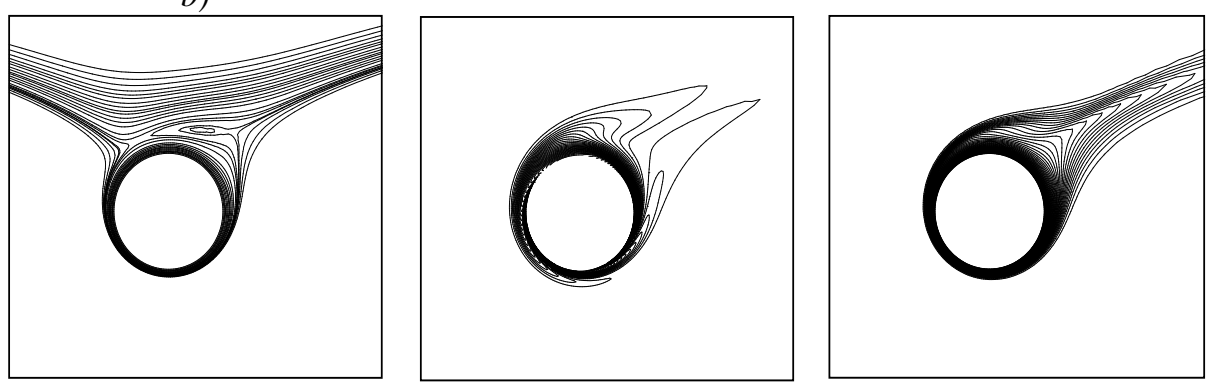

c)
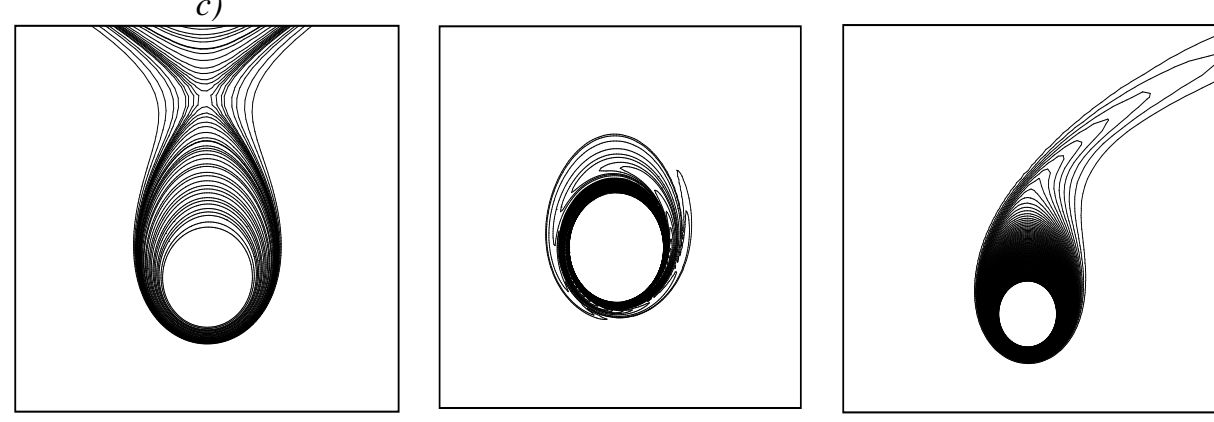

d)

Fig. 12. The streamlines, vorticity and isotherm contours around the cylinder, at a) $\alpha=1, b) \alpha=2$, c) $\alpha=3$, d) $\alpha=5$. 
The contours of positive and negative vorticity are presented in Fig. 12. The positive vorticity is generated mostly in the lower half of the surface of the cylinder while the negative vorticity is generated mostly in the upper half.

For the temperature distribution contours, it can be observed, in increasing the value of the rotation rate, the maximum density of isotherms shifts from the front surface towards the bottom surface of the rotating cylinder. It is also observed that isotherms shift in the direction of rotation of the cylinder and become almost vertical at higher values of the rotation rate. The temperature distributions presented by way of isotherms can be used to interpret the variation in the local and average heat transfer characteristics with rotation rate.

\section{Conclusion}

Unsteady laminar flow behind a rotating circular cylinder has been subjected to numerous experimental and computational studies. In this work, the flow over and heat transfer from a circular cylinder immersed in Newtonian fluids has been studied numerically. The flow transition map found by earlier researchers is shown here for rotation rates. The von Karman vortex street disappears when the rotation rate of the cylinder increases to $\alpha=2$. This is due to the weakening of the shear layers associated with flow in the wake. A second shedding mode is observed in the range of $4.34 \leq \alpha \leq$ 4.75 , characterized by the shedding of one counterclockwise vortex from the upper part of the cylinder. The core of the instability is identified in the advection of the positive vorticity of the base flow from the low-rear part of the cylinder to the stagnation point where it accumulates and is then shed. The average Nusselt number is found to decrease with increasing rotation rate. Heat transfer suppression due to rotation increases with increasing rotation rate. 


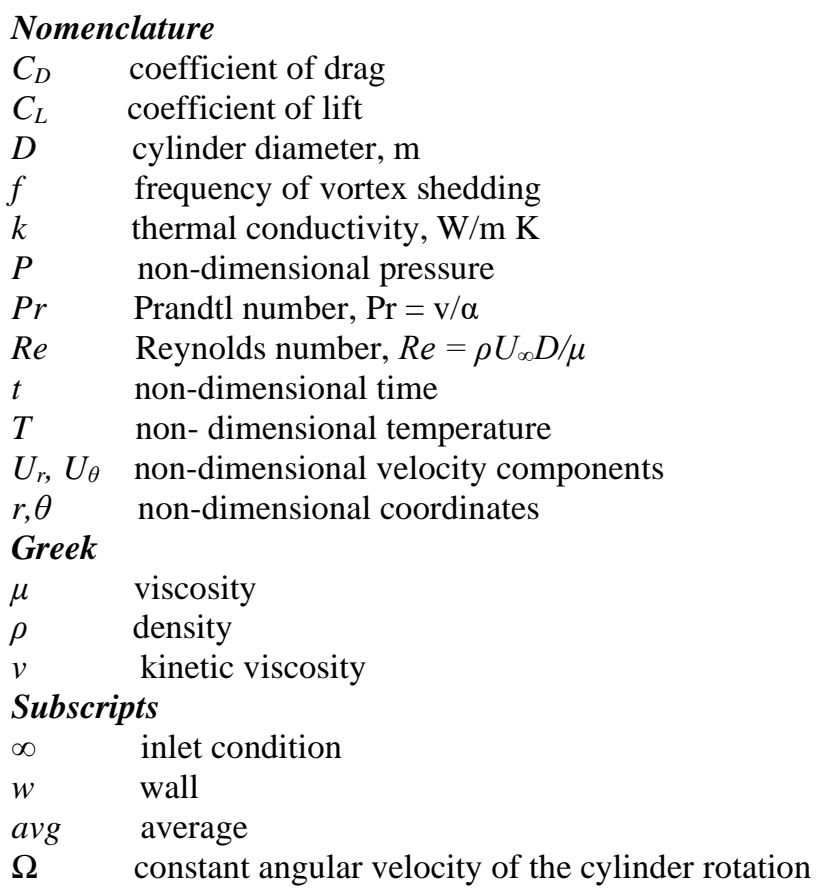

\section{References}

[1] M. Zdravkovich, Flow Around Circular Cylinders, vol. 1, Oxford Science Publication, 1997.

[2] B. N. Rajani, A. Kandasamy, S. Majumdar: Applied Mathematical Modelling, 33 (2009) 1228-1247.

[3] A. Roshko, On the development of turbulent wakes from vortex streets, Technical Report No. NACA TR 1191 (California Institute of Technology, 1953).

[4] S. Kang, H. Choi: Physics of Fluids, 11 (1999) 3312-3320.

[5] S. Mittal, B. Kumar: Journal of Fluid Mechanics, 476 (2003) 303-334.

[6] H. Nemati, M. Farhady, K. Sedighi, E. Fattahi: Thermal Sci, 3 (2010) 859-878.

[7] M.R.H. Nobari, J. Ghazanfarian: Thermal Sci, 49 (2010) 2026-2036.

[8] S.B. Paramane, A. Sharma: Int J Heat Mass Transf, 53 (2010) 4672-4683.

[9] S.B. Paramane, A. Sharma: Int J Heat Mass Transf, 52 (2009) 3205-3216.

[10] F.H. Barnes: J Phys D: Appl Phys, 33 (2000) 141-144.

[11] D. Stojkovic, P. Schon, M. Breuer, F. Durst: Phys. Fluids, 14 (2002) 3160-3178.

[12] R. El Akoury, M. Braza, R. Perrin, G. Harran, Y. Horau: J Fluid Mech, 607 (2008) $1-11$.

[13] C. Norberg: J. Fluid Mech, 258 (1994) 287-316.

[14] F.M.Mahfouz, H.M. Badr: Int. J. of Heat and Mass Transfer, 43 (2000) 3093-3104.

[15] S. Taneda: J Phys Soc Jpn, 45 (1978) 1038 -1043.

[16] C.H.K. Williamson: J Fluid Mech, 206 (1989) 579-627.

[17] H. Ding, C. Shu, K.S. Yeo, D. Xu: Int J Num Meth Fluids, 53 (2007) 305-332.

[18] I. Harimi, M. Saghafian: J Fluids Structures, 28 (2012) 309-327.

[19] A.B. Harichandan, A. Roy: Int J Heat Fluid Flow, 31 (2010) 154-171.

[20] K. Lam, W.Q. Gong, R.M.C. So: J Fluids Structures, 24 (2008) 34-57. 
[21] N. Mahir, Z. Altac: Int J Heat Fluid Flow, 29 (2008) 1309-1318.

[22] C. Norberg: Journal of Fluids Mechanics, 258 (1994) 287-316.

[23] D. Stojkovic, P. Schon, M. Breuer, F. Durst: Phys Fluids, 15 (2003) 1257-1260.

\section{(c) (i) Creative Commons License}

This work is licensed under a Creative Commons Attribution 4.0 International License. 